\title{
Correction to: Is minimally invasive liver surgery a reasonable option in recurrent HCC? A snapshot from the I Go MILS registry
}

\author{
Giovanni Battista Levi Sandri ${ }^{1}$ (1) Marco Colasanti ${ }^{1}$ - Luca Aldrighetti ${ }^{2}$. Alfredo Guglielmi ${ }^{3}$. Umberto Cillo ${ }^{4}$. \\ Vincenzo Mazzaferro ${ }^{5,6} \cdot$ Raffaele Dalla Valle $^{7} \cdot$ Luciano De Carlis $^{8,9} \cdot$ Salvatore Gruttadauria ${ }^{10,13}$. \\ Fabrizio Di Benedetto ${ }^{11}$. Alessandro Ferrero ${ }^{12}$. Giuseppe Maria Ettorre ${ }^{1}$ on behalf of I Go MILS Group on HCC
}

Published online: 29 October 2021

(C) Italian Society of Surgery (SIC) 2021

Correction to: Updates in Surgery

https://doi.org/10.1007/s13304-021-01161-w

In the originally published article the co-author first name and last name was interchanged. The correct name is copied below

Salvatore Gruttadauria

The original article has been updated.

Publisher's Note Springer Nature remains neutral with regard to jurisdictional claims in published maps and institutional affiliations.

The original article can be found online at https://doi.org/10.1007/ s13304-021-01161-w.

Giovanni Battista Levi Sandri

gblevisandri@gmail.com

$\triangle$ Luca Aldrighetti

aldrighetti.luca@hsr.it

1 Division of General Surgery and Liver Transplantation, S. Camillo Hospital, Rome, Italy

2 Hepatobiliary Surgery, IRCCS San Raffaele Hospital, Milan, Italy

3 Department of Hepatobiliary Surgery, G.B. Rossi Hospital, University of Verona, Verona, Italy

4 Department of Surgery, Oncology and Gastroenterology, University of Padua, Padua, Italy

5 Department of Surgery, HPB Surgery and Liver Transplantation, University of Milan, Milan, Italy

6 Department of Oncology, University of Milan, Milan, Italy

7 Department of Medicine and Surgery, University of Parma, Parma, Italy
Surgical and Transplant Department, ASST Grande Ospedale Metropolitano Niguarda, Milan, Italy

9 University of Milano-Bicocca, School of Medicine, Milan, Italy

10 Department for the Treatment and Study of Abdominal Diseases and Abdominal Transplantation, Istituto di Ricovero e Cura a Carattere Scientifico-Istituto Mediterraneo per i Trapianti e Terapie ad Alta Specializzazione (IRCCS-ISMETT), University of Pittsburgh Medical Center (UPMC), 90127 Palermo, Italy

11 Hepato-Pancreato-Biliary Surgery and Liver Transplantation Unit, University of Modena and Reggio Emilia, Modena, Italy

12 Department of HPB and Digestive Surgery, Ospedale Mauriziano Umberto I, Turin, Italy

13 Department of Surgery and Medical and Surgical Specialties, University of Catania, 95124 Catania, Italy 